\title{
Construção de procedimento operacional padrão para sala de imunização
}

\author{
Construction of standard operation procedure for immunization room
}

\section{Construcción de procedimiento de funcionamiento estándar para sala de inmunización}

Camilla Cristina Lisboa do Nascimento ${ }^{1 *}$, Josiane das Graças Carvalho Oliveira ${ }^{1}$, Bruno Vinicius da Costa Silva ${ }^{1}$, Adriely Alciany Miranda dos Santos ${ }^{1}$, Joelma do Socorro de Souza Tota ${ }^{2}$, Gabriel Henrique Vasconcelos da Silva ${ }^{3}$.

\section{RESUMO}

Objetivo: Construir um Procedimento Operacional Padrão (POP) sobre imunização para equipe de enfermagem da sala de vacina. Métodos: Trata-se de um estudo metodológico, com a equipe de enfermagem da sala de vacina de uma unidade de ensino e assistência de Belém - Pará. O estudo teve dois momentos: realização do estado da arte e reuniões do Grupo de Trabalho (GT) para desenvolvimento e avaliação do POP. Por se tratar de construção de um POP, não houve necessidade de aprovação do Comitê de Ética. Resultados: A partir do GT, com 9 colaboradores da equipe de enfermagem, e do estado da arte, pode-se criar um POP dentro das necessidades organofuncional para a equipe da sala de vacina, constituído em 18 categorias que foram apontadas para a melhoria do serviço de assistência à saúde para a comunidade. Conclusão: A construção do POP para sala de vacina torna-se uma forma de baixo custo para promover a saúde na APS, além de conseguir efetivar a organização e a padronização dos atendimentos.

Palavras-chave: Protocolos de Enfermagem, Imunização, Estudos de Validação.

\begin{abstract}
Objective: Build a Standard Operation Procedure (POP) about immunization to nursing team at vaccine room. Methods: It is a methodological study with nursing team at vaccine room of a teaching and assistance health center in Belém - Pará. The study had two moments: realization the state of the art and working group (WG) mettings for development and evaluation of POP. There was no need for ethics committee approval due to be an OPP construction. Results: Through the WG with 9 contributors from nursing group, and state of the art, which was created to OPP within the organo-functional necessities to team at vaccine room. It consists of 18 categories aimed at improving the health care service for community. Conclusion: The POP construction for vaccine room becomes a low-cost way to promote health in primary health care, in addition to achieving the organization and standardization of care.
\end{abstract}

Key words: Nursing Assessment, Immunization, Validation Studies.

\section{RESUMEN}

Objetivo: Construir un Procedimiento de Funcionamiento Estándar (POP) sobre inmunización para equipo de enfermería de la sala de vacuna. Métodos: Se trata de un estudio metodológico, con el equipo de

${ }^{1}$ Universidade do Estado do Pará (UEPA), Belém-PA. *E-mail: camilla.nasc@gmail.com

2 Universidade Paulista (UNIP), Belém-PA.

${ }^{3}$ Universidade da Amazônia (UNAMA), Belém-PA. 
enfermería de la sala de vacuna de una unidad de enseñanza y asistencia de Belém - Pará. El estudio tuvo dos momentos: realización del estado del arte y reuniones del Grupo de Trabajo (GT), en el que se analizan los resultados de la investigación para el desarrollo y la evaluación del POP. Por tratarse de la construcción de un POP, no hubo necesidad de aprobación del Comité de Ética. Resultados: A partir del GT, con 9 colaboradores del equipo de enfermería, y del estado del arte, se puede crear un POP dentro de las necesidades organofuncionales para el equipo de la sala de vacuna, constituido en 18 categorías que fueron apuntadas para la mejora del desempeño servicio de asistencia a la salud para la comunidad. Conclusión: La construcción del POP para sala de vacuna se convierte en una forma de bajo costo para promover la salud en la APS, además de lograr efectivizar la organización y la estandarización de las atenciones.

Palabras clave: Evaluación en Enfermaría, Inmunización, Estudios de Validación.

\section{INTRODUÇÃO}

A Atenção Primária à Saúde (APS) é entendida como o primeiro nível do Sistema Único de Saúde (SUS) e porta de entrada preferencial, com ações resolutivas sobre os problemas de saúde, articulando-se com os demais níveis de complexidade, formando uma rede integrada de serviços (STARFIELD B, 2002).

Martins AKL et al. (2011) afirma em seus estudos que as ações da APS na comunidade caracterizam-se por práticas em níveis individuais e coletivas, realizadas e acompanhadas por equipes multiprofissionais que tem como objetivo principal a promoção da saúde, prevenção de agravos, diagnóstico, reabilitação e manutenção da saúde.

No âmbito da APS, organizam-se diversos Políticas e Programas de Saúde que fazem parte da política de atenção aos diversos grupos populacionais tais como: mulheres, crianças, homens, populações tradicionais, entre outros (BRASIL, 2012).

Nesse Contexto, o Programa Nacional de Imunização (PNI) é visto como uma das principais e mais relevantes intervenções em saúde pública no Brasil, em especial pelo importante impacto obtido na redução de doenças nas últimas décadas. Por meio dele, é organizada a Política Nacional de Vacinação da população brasileira e tem como missão o controle, a erradicação e a eliminação de doenças imunopreveníveis (BRASIL, 2014). Bispo WF et al. (2017) destacam que a imunização, por meio da vacina, é uma das práticas de saúde pública mais exitosa, que contribui para a redução da incidência das doenças imunopreveníveis no Brasil.

A partir disso, faz-se necessária a organização das normas e procedimentos das tarefas executadas dentro do PNI, para estas serem realizadas com qualidade e eficácia dentro da APS. Nesse processo, destaca-se equipe de enfermagem, que deve ser capacitada para a realização das atividades da em sala de vacina.

Essa equipe é formada por, pelo menos, um técnico ou auxiliar de enfermagem, em cada turno de trabalho, e um enfermeiro responsável pela supervisão das atividades da sala de vacina e pela educação permanente da equipe (BRASIL, 2001).

Nesse sentido, destaca-se a construção de Procedimento Operacional Padrão (POP), o qual é visto como documento organizacional que traduz o planejamento do trabalho a ser executado (GOUREVITH P e MORRIS $E$, 2008). Isto é, o POP é a instrução detalhada para alcançar a uniformidade na aplicação de uma determinada função (BARBOSA CM, et al., 2011).

Ressalta-se que o POP deve ser construído de forma detalhada para visando a padronização da assistência, visto que o mesmo se apresenta como uma descrição de todas as medidas necessárias para a realização de uma determinada tarefa (DAINESI SM e NUNES DB, 2007).

Tendo em vista o exposto, definiu-se como objetivo do estudo construir um POP de imunização para equipe de enfermagem da sala de vacina, garantindo a qualidade no atendimento e efetivação do fluxo da mesma. 


\section{MÉTODOS}

Trata-se de um estudo metodológico, por se tratar de um estudo voltado para a inquirição de novos métodos e procedimentos adotado em um determinado espaço (LIMA DVM, 2011). Foi realizado nos meses de setembro a dezembro de 2018 na sala de vacina de uma unidade de ensino e assistência de Belém Pará.

O estudo foi realizado em dois momentos: realização do estado da arte sobre o tema e reuniões do Grupo de Trabalho (GT) para desenvolvimento do POP. Foram convidados enfermeiros, técnicos de enfermagem e acadêmicos de enfermagem em estágios extracurriculares da unidade, para compor o grupo de trabalho, responsável em analisar e colaborar com o processo da construção do produto final.

Por se tratar de construção de um POP, não houve necessidade de aprovação do Comitê de Ética. No entanto, o projeto de construção do material obteve autorização institucional para a sua realização.

No primeiro momento, foi realizado um estado da arte para conhecer os saberes e práticas da equipe de enfermagem na sala de vacina, a fim de destacar os procedimentos principais a serem inclusos no POP; considerando-se as etapas: busca na literatura; avaliação dos estudos incluídos na revisão; interpretação dos resultados; síntese do conhecimento; e organização dos pontos principais a serem inclusos no POP (BARBOSA CM, et al., 2011).

As produções científicas foram pesquisadas nas seguintes bases de dados: Literatura Latino-Americana e do Caribe em Ciências da Saúde (LILACS) e Bases de Dados de Enfermagem (BDENF). Foram utilizados descritores controlados (via Descritores em Ciências e Saúde): "Conhecimento AND Vacinação AND Enfermagem" e descritores não controlados, com o intuito de ampliar a busca nas bases escolhidas. Foram incluídos estudos experimentais e não experimentais, que abordavam sobre a temática; estudos publicados em português, disponível em texto completo, no período de janeiro de 2010 a novembro de 2018.

Foram excluídos todos os estudos secundários, como revisões de literatura, integrativas ou sistemáticas e artigos não pertinentes ao tema proposto. A busca dos artigos foi realizada no mês de dezembro de 2018.

Além disso, foram também somados ao estudo os manuais, normas e informes técnicos, sobre a temática, disponibilizados pelo Ministério da Saúde (MS) sendo estes: Manual de Normas e Procedimentos para Vacinação, Manual de Rede de Frios, Manual de Centro de Referência para Imunobiológicos Especiais e Manual de Vigilância Epidemiológica de Eventos Adversos Pós-Vacinação.

No segundo momento, foram realizadas reuniões semanais com o grupo de trabalho para avaliação do desenvolvimento do POP. Nos encontros foram realizadas discussões sobre o PNI; quantidade necessária de POP para a prática da imunização; estrutura e designer do POP; e, tipo de linguagem utilizada.

Com as informações obtidas no estado da arte, com as diretrizes do MS e avaliação crítica do grupo de trabalho, foram construídos os POPs. Para torná-lo mais didático e objetivo em sua utilização, foi empregada linguagem simples em seu conteúdo e a criação de um designer gráfico, na perspectiva de obter melhor facilidade de entendimento durante a sua leitura.

\section{RESULTADOS E DISCUSSÃO}

A construção dos POPs para a sala de vacina possibilitou organizar e padronizar o fluxo de atendimento para a imunização. A implantação dos POPs faz-se necessária para a organização dos procedimentos das tarefas executadas de acordo com a PNI, para estas serem realizadas com qualidade e eficácia dentro da unidade.

O estado da arte foi fundamental para comparar as práticas dentro do serviço com outras salas de vacina e observar quais os pontos fundamentais necessários em serem descritos no POP. Foram encontrados, 33 artigos com os descritores e, após aplicar o critério de exclusão, obteve-se 13 artigos, como destacados a seguir: 
Tabela 1 - Estudos incluídos no estado da arte. Belém-PA, 2019.

\begin{tabular}{|c|c|c|}
\hline AUTORIA/ANO & OBJETIVO & PRINCIPAIS ACHADOS \\
\hline $\begin{array}{l}\text { FEITOSA LR et al., } \\
2010\end{array}$ & $\begin{array}{l}\text { Analisar conhecimentos e práticas do } \\
\text { auxiliar de enfermagem em sala de } \\
\text { imunização, considerando seu papel } \\
\text { fundamental na Atenção Básica. }\end{array}$ & $\begin{array}{l}\text { Ressalta a não periodicidade de cursos de } \\
\text { atualização para o manuseio de } \\
\text { imunobiológicos e a falta de tempo dos } \\
\text { auxiliares, devido a dinâmica do processo } \\
\text { do trabalho, para tirar dúvidas com o } \\
\text { profissional de enfermagem, fazendo com } \\
\text { que ocorra condutas contraria do que é } \\
\text { preconizado. }\end{array}$ \\
\hline $\begin{array}{l}\text { OLIVEIRA VG et al., } \\
2010\end{array}$ & $\begin{array}{l}\text { ldentificar as ações de enfermagem na } \\
\text { sala de vacina e descrever o } \\
\text { conhecimento das mães/cuidadores } \\
\text { acerca da vacinação infantil. }\end{array}$ & $\begin{array}{l}\text { Destaca a priorização de realizar um } \\
\text { trabalho mais técnico, dentro da sala de } \\
\text { vacina, do que atividades de ação } \\
\text { educativa para os usuários ou capacitação } \\
\text { interna para a equipe de enfermagem. }\end{array}$ \\
\hline $\begin{array}{l}\text { BISETTO LHL et al. } \\
2010\end{array}$ & $\begin{array}{l}\text { Identificar eventos adversos pós- } \\
\text { vacinação, foco da } \\
\text { prática da enfermagem, em base de } \\
\text { dados do Sistema de Informação de } \\
\text { Eventos } \\
\text { Adversos Pós-Vacinação e discutir a } \\
\text { atuação do enfermeiro na sua vigilância }\end{array}$ & $\begin{array}{l}\text { Reflete sobre a importância da implantação } \\
\text { de educação continuada para a equipe de } \\
\text { enfermagem, visto que a } \\
\text { a maioria das intervenções estabelecidas } \\
\text { no manual de eventos adversos estava de } \\
\text { acordo com a literatura, porém verificaram- } \\
\text { se diferenças de conteúdo entre as } \\
\text { condutas para um mesmo evento } \\
\text { decorrente de vacinas diferentes. }\end{array}$ \\
\hline $\begin{array}{l}\text { LUNA GLM et al., } \\
2011\end{array}$ & $\begin{array}{l}\text { Caracterizar a experiência e atualizar o } \\
\text { conhecimento sobre imunização da } \\
\text { equipe de enfermagem responsável } \\
\text { pelas atividades nas salas de vacinas. }\end{array}$ & $\begin{array}{l}\text { Apresenta a falta de conhecimento da } \\
\text { equipe de enfermagem para gerenciar o } \\
\text { trabalho com imunobiológicos e a } \\
\text { necessidade de treinamento específico } \\
\text { para que se possa ofertar serviço de } \\
\text { qualidade. }\end{array}$ \\
\hline $\begin{array}{l}\text { SILVA FJCP et al., } \\
\qquad 2011\end{array}$ & $\begin{array}{l}\text { Analisar o estado vacinal e o } \\
\text { conhecimento prévio sobre o vírus da } \\
\text { hepatite B pelos profissionais de saúde } \\
\text { (PS) de um hospital público. }\end{array}$ & $\begin{array}{l}\text { Afirma a necessidade de implantar } \\
\text { programas de educação continuada, visto } \\
\text { que os profissionais com nível superior } \\
\text { mostraram mais conhecimento sobre o } \\
\text { tema em detrimento dos profissionais com } \\
\text { nível técnico. }\end{array}$ \\
\hline SILVA FP et al., 2011 & $\begin{array}{l}\text { Realizar diagnóstico das práticas de } \\
\text { educação em saúde vivenciadas no } \\
\text { atendimento em sala de vacinas }\end{array}$ & $\begin{array}{l}\text { Enfatiza que a prática de ações educativas } \\
\text { são apresenta dificuldade para a sua } \\
\text { realização, tanto em questões estruturais } \\
\text { como há o déficit de recursos humanos. } \\
\text { Além disso, destaca que as orientações à } \\
\text { clientela são realizadas por meio da } \\
\text { transmissão verticalizada } \\
\text { conhecimento. }\end{array}$ \\
\hline $\begin{array}{l}\text { ANDRADE TMF et al., } \\
2013\end{array}$ & $\begin{array}{l}\text { Avaliar o conhecimento de acadêmicos } \\
\text { de enfermagem acerca do HPV e sua } \\
\text { vacinação. }\end{array}$ & $\begin{array}{l}\text { Afirma que a maioria dos acadêmicos de } \\
\text { enfermagem, que passaram por práticas } \\
\text { curriculares em sala de vacina, não } \\
\text { apresentam conhecimento prévio sobre o } \\
\text { vírus, suas consequências, formas } \\
\text { preventivas e de tratamento, o que pode } \\
\text { influenciar negativamente para construção } \\
\text { de novos profissionais da área, além da } \\
\text { vulnerabilidade deste grupo etário. }\end{array}$ \\
\hline $\begin{array}{l}\text { SILVA-JUNIOR MF et } \\
\text { al., } 2014\end{array}$ & $\begin{array}{l}\text { Avaliar o conhecimento sobre a } \\
\text { vacinação da hepatite B, de acadêmicos } \\
\text { dos cursos de Medicina, Odontologia e } \\
\text { Enfermagem da Universidade Federal do } \\
\text { Espírito Santo (UFES). }\end{array}$ & $\begin{array}{l}\text { Reflete que, mesmo os acadêmicos } \\
\text { conheçam a importância sobre vacinação, } \\
\text { não há suficiência para fazê-los a ter a } \\
\text { caderneta atualizada. }\end{array}$ \\
\hline
\end{tabular}




\begin{tabular}{|c|c|c|}
\hline $\begin{array}{c}\text { PENTEADO MS et } \\
\text { al.,2015 }\end{array}$ & 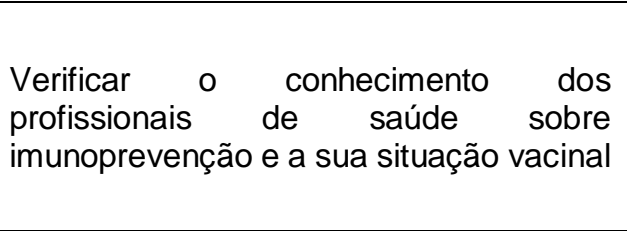 & $\begin{array}{l}\text { Mostra que os profissionais não procuram } \\
\text { manter a sua caderneta de vacinação } \\
\text { atualizada, mesmo que os mesmos } \\
\text { apresentem o conhecimento e as } \\
\text { informações sobre a importância da } \\
\text { vacinação para autoproteção. }\end{array}$ \\
\hline $\begin{array}{l}\text { ALMEIDA MG, } \\
\text { EVANGELISTA TM, } \\
2015\end{array}$ & $\begin{array}{l}\text { Avaliar conhecimentos e práticas } \\
\text { adotadas para conservação de } \\
\text { imunobiológicos por profissionais de } \\
\text { salas de vacina no Piauí. }\end{array}$ & $\begin{array}{l}\text { Destaca a importância de realizar } \\
\text { capacitação dos profissionais } \\
\text { responsáveis pela imunização, uma vez } \\
\text { que o conhecimento e a prática não são } \\
\text { considerados satisfatórios. }\end{array}$ \\
\hline $\begin{array}{c}\text { COSTA NMN, LEÃO } \\
\text { AMM, } 2015\end{array}$ & $\begin{array}{l}\text { Caracterizar a população atingida pelos } \\
\text { eventos adversos pós-vacinação - } \\
\text { segundo o sexo, idade, identificação das } \\
\text { vacinas - e analisar os eventos. }\end{array}$ & $\begin{array}{l}\text { Enfatiza que o profissional de enfermagem, } \\
\text { responsável pela sala de vacina, deve ter } \\
\text { conhecimento dos imunobiológicos e dos } \\
\text { seus eventos adversos para preveni-los. }\end{array}$ \\
\hline $\begin{array}{l}\text { MARTINS JRT et } \\
\text { al.,2017 }\end{array}$ & $\begin{array}{l}\text { Compreender, sob a ótica do profissional, } \\
\text { a Educação Permanente em sala de } \\
\text { vacina em seu contexto real. }\end{array}$ & $\begin{array}{l}\text { Destaca os que as entraves para não } \\
\text { realização de educação permanente em } \\
\text { saúde na sala de vacina são: sobrecarga } \\
\text { de trabalho associada a recursos humanos } \\
\text { insuficientes, o distanciamento do } \\
\text { enfermeiro da sala de vacina e a falta de } \\
\text { apoio das instâncias superiores. }\end{array}$ \\
\hline SILVA PMC et al,2018 & $\begin{array}{ll}\text { Desvelar o conhecimento e atitudes de } \\
\text { meninas, mães, professores e } \\
\text { profissionais da saúde sobre o } \\
\text { Papilomavírus humano e a vacinação. }\end{array}$ & $\begin{array}{l}\text { Aborda a necessidade de novas } \\
\text { estratégias educativas na comunidade e a } \\
\text { mudança de comportamento dos } \\
\text { profissionais da sala de vacina sobre a } \\
\text { orientação para os usuários, a fim de } \\
\text { garantir a imunização do público-alvo. }\end{array}$ \\
\hline
\end{tabular}

FONTE: Próprio dos autores, 2019.

Os $15 \%$ dos referenciais teóricos refletem sobre a importância da conservação de imunobiológicos. Um estudo realizado no ano de 2015 em 23 salas de vacina, de 14 municípios do Piauí, destaca que a maioria dos profissionais de enfermagem apresentam práticas inadequadas para a conservação de imunobiológicos (ALMEIDA MG e EVANGELISTA TM, 2015).

Além disso, 15\% mostram que a equipe de enfermagem da sala de imunização tem pouco conhecimento sobre a forma correta de realizar o descarte de materiais biológicos e resíduos. O PNI preconiza que o descarte do resíduo produzido na sala de vacina deve ser efetuado de maneira correta, para garantir a segurança da equipe, da população e do meio ambiente (FEITOSA LR, et al., 2010).

Isto é, $30 \%$ dos artigos discutem sobre a necessidade dos profissionais buscarem atualização dos seus conhecimentos para realizar com eficácia a prática de imunização, visto que esta é imprescindível para uma assistência de qualidade para o usuário.

Já $15 \%$ da literatura fala sobre a conduta de enfermagem frente aos eventos adversos pós-vacinação (EAPV). Uma pesquisa feita em 2011 aponta que, mesmo com as intervenções preconizadas durante o atendimento de EAPV, a maioria das condutas para um mesmo evento é diferente (BISETTO LHL, et al., 2011). Corroborando com esse pensamento, Costa NMN e Leão AMM (2015) atentam que o enfermeiro do setor deve ter conhecimento dos imunobiológicos e dos seus eventos adversos para preveni-los e saber qual tipo de conduta tomar para cada EAPV.

Todos os estudos apresentam em comum à necessidade da realização da educação permanente em saúde (EPS) com a equipe de enfermagem responsável pela imunização. O POP pode ser utilizado como forma de EPS, visto que o enfermeiro pode utiliza-lo como estratégia para ter maior proximidade com a sala de vacinação para a sua equipe (SALES CB, et al., 2018). Essa estratégia deve transcender ao tecnicismo e às capacitações pontuais, instigando a participação ativa da equipe em todo o processo (SARDINHA PL, et al., 2013). 
É notório destacar que não foram encontrados artigos que remetesse a construção, implantação ou validação de POP dentro da sala de vacina, mostrando a lacuna existente dentro desse tipo de viés de estudo.

Para a construção do POP, participaram dois enfermeiros responsáveis pela sala de vacina da unidade, em diferentes turnos; com faixa etária de 33 a 42 anos de idade e de 6 a 18 anos de profissão e 12 atuando dentro da imunização. Quanto ao grau de escolaridade, um possui mestrado e outro possui pós-graduação lato sensu.

Com relação aos outros membros da equipe de enfermagem atuantes na sala de vacinação, participaram 3 técnicos de enfermagem; com idade entre 33 e 42 anos. Possuem em média 12 anos de formação técnica e 9 anos em atuação dentro da sala de vacina.

Por se tratar de uma unidade escola, também estiveram presente no processo 3 estagiários, do curso de graduação em enfermagem, em práticas extracurriculares na unidade com idade entre 21 e 24 anos.

A estratégia de grupo de trabalho, com total de 9 colaboradores, para avaliação crítica do produto se apresentou de forma efetiva para o processo, visto que para ter bom debate ou discussão é aconselhável trabalhar com poucos indivíduos (REZENDE JL, 2003). Ressalta-se a importância da equipe de enfermagem incluída no GT, por ser a responsável em gerenciar e executar os serviços de imunização na APS, destacando-se o enfermeiro que apresenta a responsabilidade técnica e administrativa das atividades em sala de vacina, que devem ser realizadas pela equipe de enfermagem devidamente capacitada para 0 manuseio, conservação e administração dos imunobiológicos (OLIVEIRA VC, et al., 2013).

Além disso, destaca-se a inclusão dos acadêmicos de enfermagem no processo, visto a necessidade da implantação de novas metodologias de ensino visando à formação de profissionais, contemplando os princípios e as diretrizes do SUS e orientando-os às necessidades da população (GONÇALVES CB, et al., 2016). Isto é, este processo reforça o ensino-aprendizagem, ao somar o ensinamento teórico ao prático, no qual o acadêmico é aperfeiçoado em sua totalidade de forma educativa e formativa, com isso, potencializando indivíduos críticos e agentes de transformação social para realizar com excelência a prestação de serviços à comunidade (RODRIGUES MSP e LEITÃO GCM, 2000; COURY HJCG e VILELLA L, 2009).

Durante as reuniões do GTs, foi elaborada a estrutura dos POPS para padronização dos mesmos, deveria conter em seu cabeçalho: setor, número do POP, data de validação pelo GT, data de revisão, versão, elaboração, título do POP. Para a execução do procedimento, constaria: executante, objetivo, materiais necessários, descrição do procedimento, anexos e referências.

Para a sala de vacina da unidade, foram elaborados 18 POPs, a citar:

Quadro 2 - Títulos e objetivos dos Procedimentos Operacionais Padrão da Sala de Vacina. Belém-PA, 2019.

\begin{tabular}{|c|c|c|}
\hline № & TÍTULO & OBJETIVO \\
\hline 1 & LAVAGEM DAS MÃOS & $\begin{array}{l}\text { Reduzir a microbiota resistente e } \\
\text { transitória. }\end{array}$ \\
\hline 2 & ORGANIZAÇÃO DA SALA & $\begin{array}{l}\text { Padronizar a organização e funcionamento } \\
\text { da sala de imunização }\end{array}$ \\
\hline 3 & ATENDIMENTO INICIAL & $\begin{array}{l}\text { Garantir assistência adequada ao usuário } \\
\text { no procedimento de imunização }\end{array}$ \\
\hline 4 & ADMINISTRAÇÃO DE IMUNOLÓGICO & $\begin{array}{l}\text { Padronizar a administração dos } \\
\text { imunobiológicos }\end{array}$ \\
\hline 5 & ADMINISTRAÇÃO DE VACINA ORAL (VO) & $\begin{array}{l}\text { Padronizar a execução de administração } \\
\text { da VO }\end{array}$ \\
\hline 6 & $\begin{array}{l}\text { ADMINISTRAÇÃO DE VACINA } \\
\text { INTRADÉRMICA (ID) }\end{array}$ & $\begin{array}{l}\text { Padronizar a execução de administração } \\
\text { da vacina ID }\end{array}$ \\
\hline 7 & $\begin{array}{l}\text { ADMINISTRAÇÃO DE VACINA } \\
\text { SUBCUTÂNEA (SC) }\end{array}$ & $\begin{array}{l}\text { Padronizar a execução de administração } \\
\text { da vacina SC }\end{array}$ \\
\hline 8 & $\begin{array}{l}\text { ADMINISTRAÇÂO DE VACINA } \\
\text { INTRAMUSCULAR (IM) }\end{array}$ & $\begin{array}{l}\text { Padronizar a execução de administração } \\
\text { da vacina IM }\end{array}$ \\
\hline
\end{tabular}

REAS/EJCH | Vol. 11 (9) | e389 | DOI: https://doi.org/10.25248/reas.e389.2019 Página 6 de 8 


\begin{tabular}{|c|c|c|}
\hline & ENCERRAMENTO DO TRABALHO DIÁRIO & $\begin{array}{l}\text { Garantir assistência adequada ao usuário } \\
\text { no procedimento de imunização. }\end{array}$ \\
\hline 0 & LIMPEZA DA SALA DE VACINA & $\begin{array}{l}\text { Garantir a diminuição de infecções } \\
\text { cruzadas e proporcionar conforto e } \\
\text { segurança }\end{array}$ \\
\hline 1 & LIMPEZA DA CÂMARA FRIA & $\begin{array}{l}\text { Garantir a diminuição da contaminação } \\
\text { dos imunobiológicos }\end{array}$ \\
\hline 2 & $\begin{array}{l}\text { LIMPEZA DO REFRIGERADOR DAS } \\
\text { BOBINAS REUTILIZÁVEIS }\end{array}$ & $\begin{array}{l}\text { Padronizar os cuidados de limpeza e } \\
\text { ambientação das bobinas reutilizáveis }\end{array}$ \\
\hline 3 & $\begin{array}{l}\text { EVENTOS ADVERSOS PÓS-VACINAÇÃO } \\
\text { (EAPV) }\end{array}$ & $\begin{array}{l}\text { Padronizar a notificação e o } \\
\text { encaminhamento correto de todos os } \\
\text { casos de EAPV }\end{array}$ \\
\hline 4 & $\begin{array}{l}\text { DESCARTE DE MATERIAIS BIOLÓGICOS E } \\
\text { RESÍDUOS }\end{array}$ & $\begin{array}{l}\text { Padronizar o descarte de lixo comum, } \\
\text { materiais biológicos e não biológicos e } \\
\text { materiais perfurocortante da sala de } \\
\text { vacinas }\end{array}$ \\
\hline 5 & ORGANIZAÇÃO DE CAMPANHAS & $\begin{array}{l}\text { Padronizar a organização das campanhas } \\
\text { vacinas }\end{array}$ \\
\hline 6 & SOLICITAÇÃO DE VACINAS & $\begin{array}{l}\text { Padronizar a solicitação de } \\
\text { imunobiológicos }\end{array}$ \\
\hline 7 & RECEBIMENTO DE NOVAS VACINAS & $\begin{array}{l}\text { Padronizar o recebimento de } \\
\text { imunobiológicos }\end{array}$ \\
\hline 8 & $\begin{array}{l}\text { PLANO DE CONTIGÊNCIA PARA } \\
\text { IMUNOBIOLÓGICOS EM CASO DE QUEDA } \\
\text { DE ENERGIA }\end{array}$ & $\begin{array}{l}\text { Garantir que não ocorram perdas físicas } \\
\text { dos imunobiológicos devo a queda de } \\
\text { energia }\end{array}$ \\
\hline
\end{tabular}

Os POPs descritos perpassam pelo cotidiano da sala de vacina, visto a necessidade de garantir uma assistência de qualidade seguindo o que é preconizado pelo PNI. Os POPs contemplaram tanto o trabalho individual quanto o coletivo, além de abordar o fluxograma dos trabalhos que vão além do atendimento ao usuário.

Com a utilização desse material conjunto a EPS, espera-se minimizar os EAPV, além de potencializar uma melhor assistência dentro da sala de vacina.

\section{CONSIDERAÇÕES FINAIS}

Os cuidados baseados em protocolos são utilizados como forma de facilitar e padronizar a prática profissional da enfermagem. Com isso, a construção do POP para sala de vacina torna-se uma forma de baixo custo para promover a saúde na APS, além de contribuir para diminuição de EAPV e perdas físicas de imunobiológicos.

\section{REFERÊNCIAS}

1. ALMEIDA MG, et al. Conhecimento e prática de profissionais sobre conservação de vacinas. Revista Enfermagem Cuidado é Fundamental, 2015; 7: 201-203.

2. BARBOSA CM, et al. A importância dos procedimentos operacionais padrão (POPs) para os centros de pesquisa clínica. Revista da Associação Médica Brasileira, 2011; 57(2): 134-135.

3. BISPO WF, et al. Relato de experiência: atualização do cartão vacinal de educadores infantis. Revista de Enfermagem UFPE online, 2017; 11(6): 2628-2637.

4. BISETTO LHL, et al. A prática da enfermagem frente aos eventos adversos pós-vacinação. Revista da Escola de Enfermagem USP, 2011; 45(5): 1128-34.

5. BRASIL. Ministério da Saúde. Secretaria de Vigilância em Saúde. Manual de procedimento para vacinação. 4nd ed. Brasília: Ministério da Saúde; 2001; 316p. 
6. BRASIL. Ministério da Saúde. Secretaria de Atenção à Saúde. Departamento de Atenção Básica. Política Nacional de Atenção Básica. 1nd ed. Brasília: Ministério da Saúde, 2012; 114p.

7. BRASIL. Ministério da Saúde. Manual de Normas e Procedimentos para Vacinação. 1nd ed. Brasília: Ministério da Saúde, 2014; 178p.

8. COSTA NMN, LEÃO AMM. Casos notificados de eventos adversos pós-vacinação: contribuição para o cuidar em Enfermagem. Revista de enfermagem UERJ, 2015; 23(3): 297-303.

9. COURY HJCG, VILELLA L. Perfil do pesquisador fisioterapeuta brasileiro. Revista Brasileira de Fisioterapia, 2009; 13(4): 356-63.

10. DAINESI SM, NUNES, DB. Procedimentos operacionais padronizados e o gerenciamento de qualidade em centros de pesquisa. Revista de Associação Médica Brasileira, 2007; 53(1): 1-12.

11. FEITOSA LR, et al. Conhecimentos e práticas do auxiliar de enfermagem em sala de imunização. Revista Cogitare Enfermagem, 2010; 5(4): 695-701.

12. GONÇALVES CB, et al. Projeto de vivências e estágios na saúde pública: perspectiva de acadêmicos de enfermagem. Revista de Enfermagem UFPI, 2016; 5(4): 80-4.

13. GOUREVITCH P, MORRIS E. Procedimento Operacional Padrão: Uma história de guerra. São Paulo: Companhia das Letras, 2008; 292p.

14. LIMA DVM. Desenhos de pesquisa: uma contribuição para autores. Online Brazilian Journal of Nursing (ONLINE), $2011 ; 10(2): 1-14$.

15. MARTINS AKL, et al. Literatura de cordel: tecnologia de educação para saúde e enfermagem. Revista de enfermagem, 2011; 19(2): 324-329.

16. OLIVEIRA VC, et al. Supervisão de Enfermagem em Sala de Vacina: A Percepção do Enfermeiro. Revista Contexto Enfermagem, 2013; 22(4): 1015-21.

17. REZENDE JL. Aplicando Técnicas de Conversação para a Facilitação de Debates no Ambiente AulaNet. Dissertação (Mestrado em Informática) - Departamento de Informática da PUC-Rio. Universidade Católica do Rio de Janeiro, Rio de Janeiro, 2003; 170p.

18. RODRIGUES MSP, LEITÃO GCM. Estágio curricular supervisionado com ênfase no desenvolvimento da autonomia e da responsabilidade. Revista Texto contexto-enfermagem, 2000; 9(3): 216-229.

19. SALES CB, et al. Protocolos Operacionais Padrão na prática profissional da enfermagem: utilização, fragilidades e potencialidades. Revista Brasileira de Enfermagem, 2018; 71(1): 138-46.

20. SARDINHA PL, et al. Educação permanente, continuada e em serviço: desvendando seus conceitos. Enfermería Global, 2013; 29: 324-340.

21. STARFIELD, B. Atenção Primária: Equilíbrio entre necessidades de saúde, serviços e tecnologias. 1nd ed. Brasília: Ministério da Saúde, 2002; 710p. 